\title{
Preface: Springer computing journal
}

\author{
Donald F. Ferguson ${ }^{1} \cdot$ Tim A. Majchrzak $^{2}$ - Ernesto Damiani ${ }^{3}$
}

Published online: 27 January 2020

(c) Springer-Verlag GmbH Austria, part of Springer Nature 2020

The papers included in this special issue of the Springer Computing Journal seek to highlight the Advances in Cloud Computing, Web Information Systems and Software for industry and services, in addition to academic applications. This special issue is the result of a combination of selected papers from the 7th International Conference on Cloud Computing and Services Science (CLOSER 2017), the 13th International Conference on Web Information Systems and Technologies (WEBIST 2017) and the 12th International Conference on Evaluation of Novel Approaches to Software Engineering (ENASE 2017).

This special issue presents four research papers with novel concepts and applications in the aforementioned areas. Each paper was reviewed by at least two experts and the final selection reflects thorough revisions.

This special issue includes the contributions "Method, Formalization, and Algorithms to Split Topology Models for Distributed Cloud Application Deployments" by Karoline Saatkamp, Uwe Breitenbücher, Oliver Kopp and Frank Leymann, "Interpretation and Automatic Integration of Geospatial Data into the Semantic Web" by Claire Prudhomme, Timo Homburg, Jean-Jacques Ponciano, Frank Boochs, Christophe Cruz and Ana-Maria Roxin, "A Serendipity-Oriented Greedy Algorithm and an Investigation of Serendipity in Recommender Systems" by Denis Kotkov, Jari Veijalainen and Shuaiqiang Wang, and "Effective clustering protocol based on Network Division for Heterogeneous Wireless Sensor Networks" by Wided Abidi.

We hope that all papers published in this special issue help to stimulate research in the respective communities. Moreover, we are confident that the special issue underlines how conference discussions can be advanced to journal articles.

We would like to express our thanks to all the authors for their contributions and to the reviewers who helped us with their expertise and valuable time. Moreover, a

Tim A. Majchrzak

timam@uia.no

1 Columbia University, New York, USA

2 Department of Information Systems, Faculty of Social Sciences, University of Agder, Kristiansand, Norway

3 Computer Science Department, Università degli Studi di Milano, Via Giovanni Celoria, 18, 20133 Milano, Italy 
word of appreciation for their hard work goes to the Springer staff and the Computing editors, in particular to Linda Xavier, Schahram Dustdar and Christine Kamper. Finally, we thank Carla Mota, Andreia Pereira, Vitor Pedrosa and INSTICC for their arduous support in all activities revolving around CLOSER, WEBIST and ENASE. A special issue of this level is a task that can only be achieved by the collaborative effort of a dedicated and highly capable team.

We hope you enjoy this special issue.

December 2019.

Donald Ferguson

Tim A. Majchrzak

Ernesto Damiani. 